\title{
A rare localization of tuberculosis under infliximab treatment: Testicular involvement
}

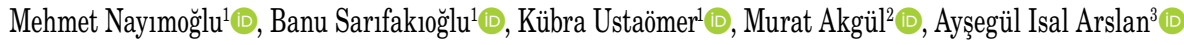 \\ Department of Physical Medicine and Rehabilitation, Namık Kemal University, School of Medicine, Tekirdağ, Turkey \\ Department of Urology, Namık Kemal University, School of Medicine, Tekirdağ, Turkey \\ Department of Pathology, Namık Kemal University, School of Medicine, Tekirdağ, Turkey
}

Received: August 06, 2019 Accepted: December 27, 2019 Published online: September 01, 2021

\begin{abstract}
Infliximab is an inhibitory of tumor necrosis factor-alpha which is used successfully for the treatment of inflammatory bowel disease and rheumatic disease. It has various side effects including injection-induced reactions, immunosuppression, demyelinating diseases, and cardiac effects. One of the most serious side effects is tuberculosis. In particular, the immunosuppressant drugs have a high risk of reactivating latent tuberculosis infection. Its activation probably may occur as an extra-pulmonary and, occasionally, may result in an unusual infection. Herein, we report a 30-year-old male case treated with infliximab and suffered from isolated testicular tuberculosis.
\end{abstract}

Keywords: Infliximab, testicular tuberculosis, tumor necrosis factor-alpha.

Infliximab is an anti-tumor necrosis factor-alfa (anti-TNF- $\alpha$ ) that has been commonly used in rheumatic diseases for many years. It has serious adverse effects such as severe infections, malignancy, gastrointestinal intolerance, hepatotoxicity ${ }^{[1]}$ The TNF- $\alpha$ is a proinflammatory cytokine produced by macrophages and other inflammatory cells. The inhibition of TNF- $\alpha$ results in the interruption of TNF receptor-mediated functions including cell activation and proliferation, production of cytokines and chemokines, and formation and maintenance of granulomas. ${ }^{[2]}$ The lack of TNF- $\alpha$ may cause granulomatous infections. ${ }^{[3]}$

Infection with Mycobacterium tuberculosis is initially contained by host defenses in the great majority of immunocompetent individuals, resulting in latent tuberculosis (TB) infection (LTBI). It remains silent for years and, in suitable conditions such as diabetes mellitus, acquired immunodeficiency syndrome, immunosuppressant therapy, and chronic kidney disease, it may be activated ${ }^{[4]}$ It usually manifests as extra-pulmonary TB. In a lower number of patients, it may occur extraordinary parts of body, such as vertebral and genitourinary involvement.

In this article, we report a 30-year-old male case with isolated testicular TB under infliximab treatment for ankylosing spondylitis (AS) and Crohn's disease (CD).

\section{CASE REPORT}

A 30-year-old male patient with AS and CD was admitted with an enlarged right testicle during routine control. The patient first realized the mass two weeks ago and it was gradually enlarged. He was diagnosed with AS and CD 10 years previously. He was administered adalimumab eight years ago. The Mantoux test result was $11 \mathrm{~mm}$ and the patient received isoniazid prophylaxis for nine months on a regular basis. Infliximab treatment was initiated

Corresponding author: Mehmet Nayımoğlu, MD. Namık Kemal Üniversitesi Tıp Fakültesi, Fiziksel Tıp ve Rehabilitasyon Anabilim Dalı, 59030 Tekirdağ, Türkiye.

e-mail: drmehmetnayimoglu@gmail.com 


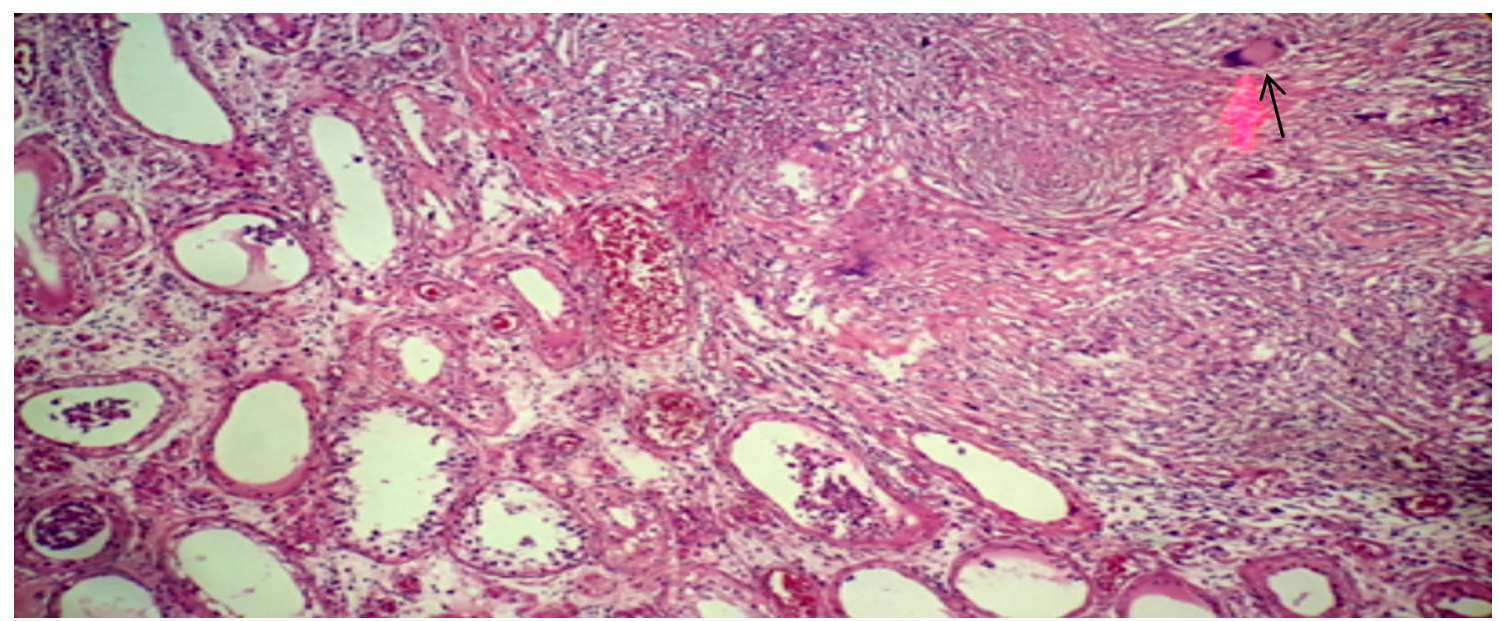

Figure 1. Intense necrotizing granulomatous inflammation in fibrous stroma and Langhans giant cells that destroy Sertoli cells in HE-stained sections $(\mathrm{H}-\mathrm{E}, \times 10)$.

HE: Hematoxylin-eosin.

due to secondary unresponsiveness after three years. Up to the present, the patient under infliximab treatment showed no signs of activation. His family history was unremarkable and rheumatologic and systemic examination findings were all normal. There were no weight loss and night sweats. Erythrocyte sedimentation rate $(79 \mathrm{~mm} / \mathrm{h})$ and C-reactive protein $(35.7 \mathrm{mg} / \mathrm{dL})$ and urinalysis results were normal. Testicular and urinary ultrasound (US) examinations revealed multiple hypoechoic lesions in the right testicular parenchyma and Doppler US revealed internal vascularization and an infiltration in the epididymis. Urinary US findings were normal. The patient was consulted to the urology department and underwent excisional biopsy with a preliminary diagnosis of lymphoproliferative disease. A written informed consent was obtained from the patient. Testicular excisional biopsy was consistent with necrotizing granulomatous inflammation (Figure 1). Upon the biopsy results, tissue and urine cultures and Ehrlich-Ziehl-Neelsen staining in the urine were performed. There was no colony in the urine culture test, while the Mycobacterium tuberculosis complex production was observed in the testicular tissue culture.

Pulmonary TB is ruled out due to the lack of respiratory symptoms with normal chest radiography and bronchoscopy findings. When the patient was questioned in terms of the source of transmission, there was no contact with any patient infected with TB and no suspicious sexual intercourse. Post diagnosis, infliximab treatment has been ceased and administered first-line anti-TB agents (isoniazid, rifampin, ethambutol, pyrazinamid). Post treatment, a significant shrinkage has occured on the testicular mass in US examination.

\section{DISCUSSION}

Infliximab is chimeric, mouse-human monoclonal antibody, used since 1999 in Europe. It is a well-known drug with favorable outcomes and is used for diseases such as spondyloarthropathies, rheumatoid arthritis, and inflammatory bowel diseases. ${ }^{[1,5]}$ Although infliximab is a drug which maintains disease control effectively, it has various side effects. The most common side effects are infection, infusion reactions, and lupus-like syndrome. Different types of infections, such as bacterial, mycobacteria, invasive fungal, viral and parasitic, have been also reported, since the time of approval of infliximab. ${ }^{[6]}$

Anti-TNF-a monoclonal antibodies, such as adalimumab and infliximab, are associated with a higher risk of $\mathrm{TB}$ than soluble TNF- $\alpha$ receptor therapy (etanercept). According to the United States Food and Drug Administration (FDA) Adverse Event Reporting System from 1998 to 2002, the estimated TB rate was $54 / 100,000$ in patients receiving infliximab. During the study period, the overall incidence of $\mathrm{TB}$ in the United States was $5.8 / 100,000 .^{[7]}$

The most common form of TB is extra-pulmonary $\mathrm{TB}$, and lymph nodes are the most commonly 
involved sites in extra-pulmonary TB. Urinary TB is uncommon and isolated testicular TB is further rare. In recent case studies regarding genitourinary $\mathrm{TB}$, the patients are from low-income countries, particularly Sub-Saharan Africa and those receiving immunosuppressive regiments due to human immunodeficiency virus infection. ${ }^{[8]}$ Another case report with rare location is endometrial TB from Africa as the former in a 72-year-old female patient with sickle cell hemoglobinopathy. ${ }^{[9]}$ Our case had testicular TB with epididymal involvement under treatment of anti-TNF- $\alpha$. However, review of the literature reveals no testicular TB case in patients treated with infliximab or other anti-TNF- $\alpha$ agents.

Tuberculosis orchitis usually occurs with TB involvement of the lower and upper urinary tract, or even of the kidneys. Therefore, it presents lower urinary tract symptoms such as irritative voiding, and hematuria. However, the present case had no symptoms of the involvement of the lower urinary tract. Epididymitis, prostatitis, scrotal swelling, and ulcerations are the other manifestations. ${ }^{[10,11]}$ Differential diagnosis of scrotal swelling is acute infection, tumor, and granulomatous inflammation. Particularly, if the scrotal swelling is the first symptom of the patient, it may be misdiagnosed as testicular cancer. ${ }^{[12]}$ Despite an eristic issue that the relation between malignancy and infliximab, it should be kept in mind that it may be relevant to infliximab. In such cases, the patient is scheduled for orchiectomy and $\mathrm{TB}$ orchitis can be diagnosed.

Although TB orchitis is usually unilateral, $12.5 \%$ of cases are bilateral. ${ }^{[13]}$ Even if the urine test result is negative for acid-resistant bacteria, it is diagnosed by urine culture or excisional tissue biopsy. ${ }^{[14]}$ In our case, as the urine culture was sterile, the diagnosis was made based on the excisional tissue biopsy. In general, testicular involvement is due to the hematogenous spread of active pulmonary TB. ${ }^{[15]}$ However, our case had no active TB, but the use of immunosuppressive therapy could explain the spread of latent TB. Another source of testicular TB is a suspicious sexual contact which may cause ulceration and granulomas on the penis. ${ }^{[15]}$ Yet, our case had a neither suspicious sexual contact, nor penile ulceration.

A meta-analysis showed that the preventive treatment decreased the TB risk by $65 \%$ in patients who received prophylaxis, compared to those who did not. ${ }^{[16]}$ Despite proper preventive treatment, the protection against LTBI is still not guaranteed.
In conclusion, anti-TNFs play an important role in controlling AS and are preferred by clinicians, as they have an ability to decelerate the disease progression. There is always a risk of LTBI, due to its immunosuppressive effect, and forms of LTBI may show atypical clinic progression and involve unusual parts of the body. Therefore, a close follow-up, detailed medical history, and physical examination can provide information and be a guide for early diagnosis and treatment of a possible TB infection in patients receiving immunosuppressive therapy.

\section{Declaration of conflicting interests}

The authors declared no conflicts of interest with respect to the authorship and/or publication of this article.

Funding

The authors received no financial support for the research and/or authorship of this article.

\section{REFERENCES}

1. Keane J. TNF-blocking agents and tuberculosis: new drugs illuminate an old topic. Rheumatology (Oxford) 2005;44:714-20.

2. Beenhouwer D, Wallis R, Broder M, Furst DE. Mechanisms of action of tumor necrosis factor antagonist and granulomatous infections. J Rheumatol 2004;31:1888-92.

3. PAY S. Romatizmal Hastalıklarda Anti-TNF ilaç kullanımı. Türkiye Klinikleri Tip Bilimleri Dergisi 2006;26:430-40.

4. Drudi FM, Laghi A, Iannicelli E, Di Nardo R, Occhiato R, Poggi R, et al. Tubercular epididymitis and orchitis: US patterns. Eur Radiol 1997;7:1076-8.

5. Wallis RS, Ehlers S. Tumor necrosis factor and granuloma biology: explaining the differential infection risk of etanercept and infliximab. Semin Arthritis Rheum 2005;34(5 Suppl1):34-8.

6. Scheinfeld N. A comprehensive review and evaluation of the side effects of the tumor necrosis factor alpha blockers etanercept, infliximab and adalimumab. J Dermatolog Treat 2004;15:280-94.

7. Wallis RS, Broder M, WongJ, Beenhouwer D. Granulomatous infections due to tumor necrosis factor blockade: correction. Clin Infect Dis 2004;39:1254-5.

8. Hane J, Duffey B, Kaiser R, Walker PF, Alpern JD. Case report: Epididymo-orchitis due to mycobacterium tuberculosis. Am J Trop Med Hyg 2019;101:1070-2.

9. Agbodande AK, Dodo RL, Issa A, Adjadohoun S, AzonKouanou A, Wanvoegbe AF, et al. Rare location of tuberculosis: endometrial tuberculosis. Pan Afr Med J 2019;33:45

10. Garbyal RS, Gupta P, Kumar S; Anshu. Diagnosis of isolated tuberculous orchitis by fine-needle aspiration cytology. Diagn Cytopathol 2006;34:698-700.

11. Shugaba AI, Rabiu AM, Uzokwe C, Matthew RM. Tuberculosis of the testis: a case report. Clin Med Insights Case Rep 2012;5:169-72. 
12. Salvador R, Vilana R, Bargalló X, Araque X, Nicolau C. Tuberculous epididymo-orchitis after intravesical BCG therapy for superficial bladder carcinoma: sonographic findings. J Ultrasound Med 2007;26:671-4.

13. Viswaroop BS, Kekre N, Gopalakrishnan G. Isolated tuberculous epididymitis: a review of forty cases. J Postgrad Med 2005;51:109-11.
14. Beacock CJ, Lynch TH, Hughes MA. Fatal tuberculous meningitis complicating tuberculous epididymitis. $\mathrm{Br} \mathrm{J}$ Urol 1991;67:328-9.

15. Wise GJ, Shteynshlyuger A. An update on lower urinary tract tuberculosis. Curr Urol Rep 2008;9:305-13.

16. Andrew OT, Schoenfeld PY, Hopewell PC, Humphreys MH. Tuberculosis in patients with end-stage renal disease. Am J Med 1980;68:59-65. 